\title{
Dietary patterns and schizophrenia: a comparison with healthy controls
}

\author{
This article was published in the following Dove Press journal: \\ Neuropsychiatric Disease and Treatment \\ 22 April 2015 \\ Number of times this article has been viewed
}

\author{
Koji Tsuruga' \\ Norio Sugawara' \\ Yasushi Sato' \\ Manabu Saito' \\ Hanako Furukori ${ }^{2}$ \\ Taku Nakagami ${ }^{3}$ \\ Kazuhiko Nakamura' \\ Ippei Takahashi ${ }^{4}$ \\ Shigeyuki Nakaji ${ }^{4}$ \\ Norio Yasui-Furukori' \\ 'Department of Neuropsychiatry, \\ Hirosaki University School \\ of Medicine, Hirosaki, Japan; \\ ${ }^{2}$ Department of Psychiatry, Kuroishi- \\ Akebono Hospital, Kuroishi, Japan; \\ ${ }^{3}$ Department of Neuropsychiatry, \\ Odate Municipal General Hospital, \\ Odate, Japan; ${ }^{4}$ Department of Social \\ Medicine, Hirosaki University School \\ of Medicine, Hirosaki, Japan
}

\begin{abstract}
Background: It has been reported that the onset of schizophrenia and the physical complications after its onset are related to diet. Diet has been considered as a variable factor of the pathogenesis of schizophrenia. However, the results of studies on this relationship have been inconsistent. Nutrients are consumed as a mixture in the diet. It is difficult to study them in isolation because they may have mutually complementary effects. The aim of this study was to assess the association between dietary patterns and schizophrenia in Japan.
\end{abstract}

Methods: The subjects comprised 237 outpatients aged 30-60 years (123 males and 114 females) with diagnoses of either schizophrenia or schizoaffective disorder. The patient diagnoses were determined based on medical records. Patients were recruited between June 2011 and August 2011. As a reference group, 404 healthy volunteers aged 30-60 years (158 males and 246 females) were also included. Demographic data (age, sex, and level of education) were collected by face-to-face method interviews and self-administered questionnaires. We assessed eating habits over the last month using a validated brief self-administered diet history questionnaire. We detected dietary patterns through a principal component analysis of calorie-adjusted intake; two principal components were retained. The principal components for each dietary pattern and for each individual were divided into tertiles by principal component scores.

Results: We derived two dietary patterns by principal component analysis; namely, the "vegetable" dietary pattern and the "cereal" dietary pattern. In the "cereal" dietary pattern, the high tertile was associated with a significantly increased risk of schizophrenia $(P<0.001)$.

Conclusion: The "cereal" dietary pattern is associated with schizophrenia. This article is the first to describe a study examining the association of dietary pattern and schizophrenia.

Keywords: schizophrenia, dietary pattern, diet

\section{Introduction}

Schizophrenia is a severe mental illness that affects approximately $1 \%$ of the population worldwide. ${ }^{1}$ Nakane et $\mathrm{al}^{2}$ reported that prevalence rates for schizophrenia in Japan ranged from $0.19 \%$ to $1.79 \%$ and morbidity risk rates ranged from $0.35 \%$ to $2.48 \%$. They remarked that when compared with mean values from other countries, no significant differences were observed. ${ }^{2}$ Unsatisfactory outcomes after pharmacotherapy ${ }^{3}$ and physical complications in patients with schizophrenia ${ }^{4}$ suggest that the dopamine hypothesis and antipsychotic treatment are not sufficient approaches to understanding the etiology of schizophrenia.

Diet has been considered as a variable factor of the pathogenesis of schizophrenia. Previous studies have shown that physical complications and schizophrenia outcomes are related to diet. ${ }^{5}$ Simonelli-Muñoz et $\mathrm{al}^{6}$ showed that patients who have unhealthy dietary habits have a higher risk of obesity than patients with good dietary habits.
Correspondence: Koji Tsuruga Department of Neuropsychiatry, Hirosaki University School of Medicine, 5 Zaifu-cho Hirosaki City, Aomori 036-8562, Japan

Tel +8I I72 395066

Fax $+8 I I 72345067$

Email cranehappiness@yahoo.co.jp
Neuropsychiatric Disease and Treatment 2015:I I III5-II 20 
Sugawara et $\mathrm{al}^{7}$ suggested that dietary patterns (including a higher intake of protein, fat, n-3 polyunsaturated fatty acids, n- 6 polyunsaturated fatty acids, and vitamins) may be related to a decreased prevalence of obesity in patients with schizophrenia. In addition, based on an ecological analysis of nutritional patterns, Peet $^{8}$ determined that a higher national dietary intake of refined sugar and dairy products predicted a worse 2-year outcome of schizophrenia.

Nutrients are consumed as a mixture in the diet. It is difficult to study them in isolation because they may have mutually complementary effects. With regard to the risk of schizophrenia, dietary patterns or combinations of foods may have a stronger association than foods or nutrients separately. We hypothesized that dietary patterns may be associated with schizophrenia.

The aim of this study was to assess the relationship between dietary patterns and schizophrenia in Japan. To our knowledge, this is the first study to examine this relationship.

\section{Methods}

\section{Participants}

The subjects comprised 237 outpatients aged 30-60 years (123 males and 114 females) diagnosed with the Diagnostic and Statistical Manual of Mental Disorders, Fourth Edition criteria of either schizophrenia or schizoaffective disorder at four psychiatric hospitals in Japan. Patient diagnoses were determined based on medical records. Patients were recruited between June 2011 and August 2011. As a reference group, 404 healthy volunteers aged 30-60 years (158 males and 246 females) who participated in the Iwaki Health Promotion Project in 2011 were also included. The study was approved by the Ethics Committee of the Hirosaki University School of Medicine, Hirosaki, Japan, and all subjects signed informed consent. Demographic data (age, sex, and level of education) were collected by face-to-face method interviews and selfadministered questionnaires.

\section{Measurements}

We assessed eating habits over the last month using a validated brief self-administered diet history questionnaire (BDHQ) ${ }^{9}$ that consisted of inquiries concerning the consumption frequency of 56 foods and beverages and nine dishes generally consumed in Japanese populations. In order to estimate the intake of calories and of selected nutrients, we used a computer algorithm for the 56 foods and beverages of the BDHQ with the Standard Tables of Food Composition in Japan. ${ }^{10,11}$

\section{Statistical analyses}

Dietary patterns were detected through a principal component analysis. We adopted the calorie-adjusted intake that used a density method for the 52 food and beverage items (excluding four items that overlapped with others). We determined the number of principal components to retain by eigenvalues, the scree test, and the interpretability of the factors; two principal components were retained. The principal components for each dietary pattern and for each individual were divided into tertiles by principal component scores.

The subjects with and without schizophrenia were compared using unpaired Student's $t$-tests for continuous variables or chi-squared tests for categorical variables. We assessed trend associations across the tertile categories of each dietary pattern using linear regression analysis for continuous variables and the Cochran-Armitage trend test for categorical variables, with ordinal values ranging from 1 to 3 assigned to the tertile categories of each dietary pattern.

A logistic regression analysis was carried out to evaluate the relationship between dietary patterns and schizophrenia. The model was adjusted for age and sex. The significance level for the results obtained in the hypothesis contrast was $P<0.05$. The data analysis was performed using $\mathrm{R}$ for Windows, Version 3.0.2 (The R Foundation for Statistical Computing, Vienna, Austria).

\section{Results}

The demographic distribution of the participants is presented in Table 1. The number of subjects of monotherapy is 156 , while the number of subjects of polypharmacy (using two or more antipsychotic agents) is 95 . The subjects of polypharmacy were receiving risperidone $(\mathrm{n}=51)$, aripiprazole $(n=25)$, olanzapine $(n=21)$, blonanserin $(n=26)$, quetiapine $(n=25)$, haloperidol $(n=41)$, levomepromazine $(n=31)$, and other antipsychotic agents.

We derived two dietary patterns by principal component analysis (Table 2). The first component, which loaded green

Table I Demographic characteristics of the subjects

\begin{tabular}{llll}
\hline & $\begin{array}{l}\text { Control } \\
(\mathbf{n = 4 0 4 )}\end{array}$ & $\begin{array}{l}\text { Schizophrenia } \\
(\mathbf{n = 2 3 7 )}\end{array}$ & P-value \\
\hline Age (years) & $49.1 \pm 8.5$ & $44.1 \pm 8.4$ & $<0.000 \mathrm{I}$ \\
Sex $(\mathrm{n})$ & Male I58, & Male I23, & 0.002 \\
& Female 246 & Female II4 & \\
Height $(\mathrm{cm})$ & $162.0 \pm 8.2$ & I70.2 \pm 9.0 & 0.002 \\
Weight $(\mathrm{kg})$ & $60.5 \pm 10.9$ & $68.9 \pm 15.5$ & $<0.000 \mathrm{I}$ \\
Body mass index $\left(\mathrm{kg} / \mathrm{m}^{2}\right)$ & $23.0 \pm 3.1$ & $25.5 \pm 5.0$ & $<0.00 \mathrm{I}$ \\
\hline
\end{tabular}

Notes: The data are expressed as the mean \pm standard deviation for categorical variables. The data were analyzed using nonpaired $t$-tests for continuous variables and chi-squared tests. 
Table 2 Factor loading matrix for major dietary patterns identified by principal component analysis ${ }^{\mathrm{a}}$

\begin{tabular}{|c|c|c|}
\hline & $\begin{array}{l}\text { Vegetable dietary } \\
\text { pattern }\end{array}$ & $\begin{array}{l}\text { Cereal dietary } \\
\text { pattern }\end{array}$ \\
\hline Low-fat milk & - & - \\
\hline Milk & - & - \\
\hline Chicken & - & - \\
\hline Pork/beef & - & - \\
\hline Ham/sausage/bacon & - & - \\
\hline Liver & - & - \\
\hline Squid/octopus/shrimp/shellfish & - & - \\
\hline Small fish with bones & - & - \\
\hline Canned tuna & - & - \\
\hline Dried fish/salted fish & - & - \\
\hline Oily fish & - & - \\
\hline Lean fish & - & - \\
\hline Egg & - & - \\
\hline Tofu/atsuage ${ }^{b}$ & 0.528 & - \\
\hline Natto ${ }^{c}$ & - & - \\
\hline Potatoes & 0.491 & - \\
\hline Picked green leafy vegetables & 0.341 & - \\
\hline Other picked vegetables & - & - \\
\hline Lettuce/cabbage & 0.632 & - \\
\hline Green leafy vegetables & 0.683 & - \\
\hline Cabbage/Chinese cabbage & 0.689 & - \\
\hline Carrots/pumpkin & $0.70 \mathrm{I}$ & - \\
\hline Japanese radish/turnip & 0.652 & - \\
\hline Other root vegetables & $0.7 \mid 4$ & - \\
\hline Tomatoes & 0.413 & - \\
\hline Mushrooms & 0.621 & - \\
\hline Seaweed & 0.509 & - \\
\hline Western-type confectioneries & - & 0.477 \\
\hline Japanese-type confectioneries & - & 0.432 \\
\hline Rice crackers/rice & - & 0.354 \\
\hline Ice cream & - & 0.388 \\
\hline Citrus fruit & - & - \\
\hline $\begin{array}{l}\text { Persimmons/strawberries/ } \\
\text { kiwi fruit }\end{array}$ & 0.348 & - \\
\hline Other fruit & - & - \\
\hline Mayonnaise/dressing & 0.338 & - \\
\hline Bread & - & 0.426 \\
\hline Buckwheat noodles & - & - \\
\hline Japanese wheat noodles & - & - \\
\hline Chinese noodles & - & - \\
\hline Spaghetti and macaroni & - & - \\
\hline Green tea & - & - \\
\hline Black tea/oolong tea & - & - \\
\hline Coffee & - & - \\
\hline Cola drink/soft drink & - & - \\
\hline $100 \%$ fruit and vegetable juice & - & - \\
\hline Rice & - & -0.648 \\
\hline Miso soup & - & -0.513 \\
\hline Sake & - & - \\
\hline Beer & - & - \\
\hline Spirits & - & - \\
\hline Whiskey & - & - \\
\hline Wine & - & - \\
\hline
\end{tabular}

Notes: aFactor loading less than \pm 0.3 represented by a dash for simplicity; bdeepfried tofu; 'fermented soybeans. leafy vegetables, lettuce, cabbage, mushrooms, and fruit, was labeled the "vegetable" dietary pattern. The second component, which loaded rice, bread, and confectioneries, was labeled the "cereal" dietary pattern.

The characteristics of the dietary pattern scores categorized by tertiles are shown in Table 3 . The subjects with higher scores for the "vegetable" dietary pattern were less likely to have schizophrenia and to be male. The "vegetable" dietary pattern was positively associated with the intake of proteins, carbohydrates, fats, saturated fatty acids, monounsaturated fatty acids, polyunsaturated fatty acids, n-3 fatty acids, and n-6 fatty acids.

The "cereal" dietary pattern was positively associated with schizophrenia $(P<0.001)$. Higher scores for the "cereal" dietary pattern were positively associated with the intake of proteins, fats, saturated fatty acids, monounsaturated fatty acids, polyunsaturated fatty acids, n-3 fatty acids, and n-6 fatty acids. The "cereal" dietary pattern was negatively associated with carbohydrate intake.

The odds ratios for schizophrenia according to the tertile categories of each dietary pattern score are shown in Table 4. The "vegetable" dietary pattern was not associated with an increased risk of schizophrenia. For the "cereal" dietary pattern, the high tertile was associated with a significantly increased risk of schizophrenia.

\section{Discussion}

We designed this cross-sectional study to evaluate the relationship between dietary patterns and schizophrenia in Japan. Two dietary patterns were derived; namely, the "vegetable" and "cereal" patterns. The "vegetable" dietary pattern was characterized by a high consumption of green leafy vegetables, seaweed, potatoes, and soybean products such as tofu and natto, and positively correlated with the intake of fats and proteins. A previous study demonstrated a relationship between fatty acids and schizophrenia. ${ }^{12}$ Therefore, we hypothesized that a dietary pattern consuming a large quantity of vegetables would be associated with a lower risk of schizophrenia morbidities. However, an apparent association was not observed between the "vegetable" dietary pattern and schizophrenia. By contrast, the "cereal" dietary pattern, which was characterized by a high consumption of rice, breads, and confectioneries and positively correlated with the intake of fats and proteins, was associated with schizophrenia. In the "cereal" pattern, schizophrenia correlated to fatty acid per calories intake.

Previous studies have demonstrated a relationship between polyunsaturated fatty acids and the pathogenesis of 


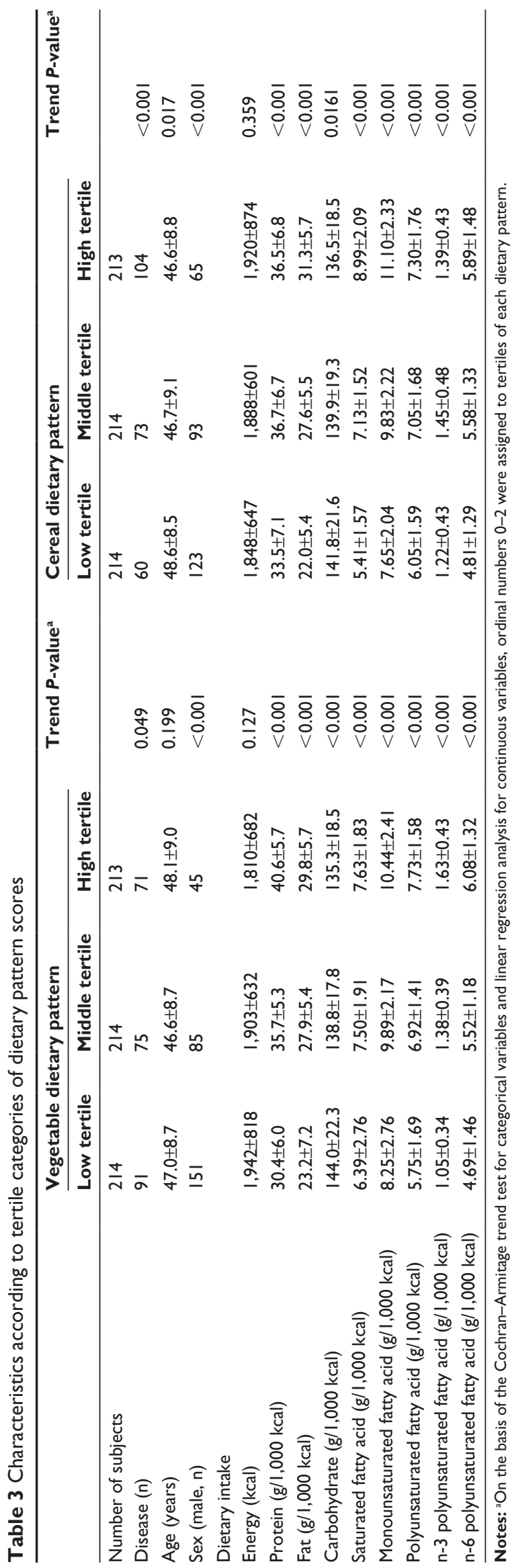

schizophrenia. ${ }^{12,13}$ A sudden change in fatty acids can play a role in a wide range of psychiatric, neurological, and developmental disorders in adults. ${ }^{12}$ A lack of omega-3 fatty acids in particular is related to the pathogenesis of schizophrenia. ${ }^{13}$ The dietary and physical activity habits of individuals with schizophrenia contribute to an unfavorable metabolic profile. The daily calories intake between patients with schizophrenia and control patients was similar; however, individuals with schizophrenia consumed significantly greater amounts of sugar and fat. ${ }^{14}$

A few studies among patients with schizophrenia also reported a significantly increased intake of calories ${ }^{15-17}$ and low consumption of both monounsaturated and polyunsaturated fatty acids. ${ }^{4}$ Five studies reported no significant difference in the diets of patients with schizophrenia compared with those of healthy subjects. ${ }^{17-21}$

Our study shows that there was a higher ratio of fatty acids in the gross calories of the diet in patients with schizophrenia. This result supports the hypothesis that fatty acids may play a role in schizophrenia pathogenesis. Alternatively, as a result of having compensated for a lack of fatty acids in the nervous system, the human body may consume more fatty acids, leading to this result.

This study also shows that patients with schizophrenia tend to consume meals that contain substantially more oil and fat. In addition, it shows that the ratio of fatty acids in the gross calories of the diet is higher in patients with schizophrenia. This result suggests that schizophrenic patients may be more likely to consume food that contains oil and fat. Roick et $\mathrm{al}^{22}$ reported that schizophrenic patients have a supper snack and consume fast food more frequently than the general population does. It is concerning that schizophrenic patients in Japan live in an environment in which a diet that contains a substantial amount of fatty acids can be obtained relatively easily and inexpensively.

Only a few studies have investigated which factors may influence the diet of individuals with schizophrenia, and the findings appear to be inconsistent. The results from two such studies suggest that the poor diet associated with schizophrenia is influenced by socioeconomic status. ${ }^{22,23}$ Fewer males, compared with the general population, reached acceptable levels for consumption of fruit, vegetables, milk, potatoes, and pulses. ${ }^{24}$ Fewer females reached the levels for consumption of milk and potatoes. ${ }^{24}$ However, other authors noted that female patients consumed more fat, carbohydrates, and overall calories ${ }^{17}$ and less fruit, vegetables, and nuts ${ }^{25}$ than females in the general population did. In any case, poor diet may be associated with schizophrenia, and the lifestyle of 
Table 4 Odds ratios and 95\% confidence intervals $(\mathrm{Cl})$ for having schizophrenia, according to tertiles of dietary pattern scores

\begin{tabular}{|c|c|c|c|c|c|}
\hline & No of cases & Crude odds ratio & $P$-value & Adjusted odds ratio ${ }^{a}$ & $P$-value \\
\hline \multicolumn{6}{|l|}{ Vegetable dietary pattern } \\
\hline Low tertile & 91 & Reference & & Reference & \\
\hline Middle tertile $(95 \% \mathrm{Cl})$ & 75 & $0.66(0.46-0.96)$ & 0.029 & $0.74(0.50-1.10)$ & 0.139 \\
\hline High tertile $(95 \% \mathrm{Cl})$ & 71 & $0.83(0.54-1.29)$ & 0.41 & $1.10(0.67-1.80)$ & 0.701 \\
\hline \multicolumn{6}{|l|}{ Cereal dietary pattern } \\
\hline Low tertile & 60 & Reference & & Reference & \\
\hline Middle tertile $(95 \% \mathrm{Cl})$ & 73 & $1.19(0.77-1.83)$ & 0.435 & $\mathrm{I} .15(0.73-1.8 \mathrm{I})$ & 0.559 \\
\hline High tertile $(95 \% \mathrm{Cl})$ & 104 & $2.43(1.67-3.56)$ & $<0.001$ & $2.71(1.80-4.09)$ & $<0.001$ \\
\hline
\end{tabular}

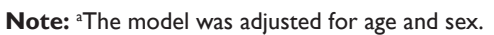

people with schizophrenia raises concerns when considering the relationship of this diet with coronary heart disease. ${ }^{24}$ In this context, limiting fat intake in patients with schizophrenia may help prevent the onset of metabolic syndrome.

This study has some limitations. First is the cross-sectional nature of the study. We cannot refer to causal determinations to be made between dietary patterns and the onset of schizophrenia. Longitudinal studies are needed to interpret these associations and causalities. Second, the dietary data were collected using the BDHQ. The results may be influenced by potential misclassification of dietary patterns, although the validity and reliability of BDHQ have been estimated. ${ }^{9,26}$ Third, we did not examine several potential confounding factors in our study, such as income levels and interpersonal relationships among families. Income levels may be a peculiarly important factor, as the results may have been confounded by low income level. Stratification studies by income levels should be requested in the future. Fourth, in this study, the ages of the participants were limited to $30-60$ years. It is known that the onset of schizophrenia commonly occurs between the ages of 10 and 20 years. Our study did not investigate the 10 to 20 -year age group; therefore, we could not accurately determine whether there was a relationship between schizophrenia and fatty acids in this younger cohort. Fifth, all of the volunteer participants may have been relatively healthier than the general population because they were more interested in their own health. Thus, the members of the community who did not participate in the study may exhibit different schizophrenia morbidities. Finally, we could not completely eliminate a beta error as the cause of our inability to detect certain associations between dietary patterns and schizophrenia, as our sample size was relatively small.

\section{Conclusion}

We hypothesized that a dietary pattern comprising high amounts of vegetables would be associated with a lower risk of schizophrenia. However, no evident association was observed between the "vegetable" dietary pattern and schizophrenia.

By contrast, the "cereal" dietary pattern, which was characterized by a high consumption of rice, bread, and confectioneries and positively correlated with the intake of fats and proteins, was associated with schizophrenia. This result may provide clues regarding the pathogenesis of schizophrenia and the prevention of metabolic syndrome in schizophrenic patients. This article is the first to describe a study of dietary patterns and schizophrenia.

\section{Acknowledgments}

The authors are grateful to the volunteers who participated in this study. The authors would like to thank all of their coworkers on this study for their skillful contributions to collecting and managing the data.

\section{Disclosure}

This study was funded by a Grant-in-Aid for Scientific Research (KAKENHI) from the Japan Society for the Promotion of Research (JSPS 20333734), Mitsubishi Pharma Research Foundation, and Asteras Schizophrenia Research Foundation and a grant from the Hirosaki Research Institute for Neurosciences. Norio Yasui-Furukori has received grant/ research support or honoraria from, and been a speaker for Asteras, Dainippon, Eli Lilly, GSK, Janssen-Pharma, Meiji, Mochida, MSD, Otsuka, Pfizer, Takada, and Yoshitomi. The remaining authors declare that they have no competing interests. The funders had no role in study design, data collection and analysis, decision to publish, or preparation of the manuscript.

\section{References}

1. van Os J, Kapur S. Schizophrenia. Lancet. 2009;374(9690):635-645.

2. Nakane Y, Ohta Y, Radford MH. Epidemiological studies of schizophrenia in Japan. Schizophr Bull. 1992;18(1):75-84.

3. Miyamoto S, Jarskog LF, Fleischhacker WW. New therapeutic approaches for treatment-resistant schizophrenia: a look to the future. J Psychiatr Res. 2014;58C:1-6. 
4. Henderson DC, Borba CP, Daley TB, et al. Dietary intake profile of patients with schizophrenia. Ann Clin Psychiatry. 2006;18(2): 99-105.

5. Peet M. Diet, diabetes and schizophrenia: review and hypothesis. $\mathrm{Br} J$ Psychiatry. 2004;184 Suppl 47:S102-S105.

6. Simonelli-Muñoz AJ, Fortea MI, Salorio P, Gallego-Gomez JI, Sánchez-Bautista S, Balanza S. Dietary habits of patients with schizophrenia: a self-reported questionnaire survey. Int J Ment Health Nurs. 2012;21(3):220-228.

7. Sugawara N, Yasui-Furukori N, Sato Y, et al. Dietary patterns are associated with obesity in Japanese patients with schizophrenia. BMC Psychiatry. 2014;14:184.

8. Peet M. International variations in the outcome of schizophrenia and the prevalence of depression in relation to national dietary practices: an ecological analysis. Br J Psychiatry. 2004;(184):404-408.

9. Kobayashi S, Murakami K, Sasaki S, et al. Comparison of relative validity of food group intakes estimated by comprehensive and brief-type self-administered diet history questionnaires against $16 \mathrm{~d}$ dietary records in Japanese adults. Public Health Nutr. 2011;14(7):1200-1211.

10. Science and Technology Agency. [Standard Tables of Food Composition in Japan]. 5th rev ed. Tokyo: Printng Bureau of the Ministry of Finance; 2005. Japanese.

11. Science and Technology Agency. [Standard Tables of Food Composition in Japan: Fatty Acid Section]. 5th rev and enlarged ed. Tokyo: Printng Bureau of the Ministry of Finance; 2005. Japanese.

12. Perica MM, Delas I. Essential fatty acids and psychiatric disorders. Nutr Clin Pract. 2011;26(4):409-425.

13. Peet M. Omega-3 polyunsaturated fatty acids in the treatment of schizophrenia. Isr J Psychiatry Relat Sci. 2008;45(1):19-25.

14. Ratliff JC, Palmese LB, Reutenauer EL, Liskov E, Grilo CM, Tek C. The effect of dietary and physical activity pattern on metabolic profile in individuals with schizophrenia: a cross-sectional study. Compr Psychiatry. 2012;53(7):1028-1033.

15. Dipasquale S, Pariante CM, Dazzan P, Aguglia E, McGuire P, Mondelli V. The dietary pattern of patients with schizophrenia: a systematic review. J Psychiatr Res. 2013;47(2):197-207.
16. DeMyer MK, Ward SD, Lintzenich J. Comparison of macronutrients in the diets of psychotic and normal children. Arch Gen Psychiatry. 1968;18(5):584-590.

17. Strassnig M, Brar JS, Ganguli R. Nutritional assessment of patients with schizophrenia: a preliminary study. Schizophr Bull. 2003;29(2): 393-397.

18. Blouin M, Tremblay A, Jalbert ME, et al. Adiposity and eating behaviors in patients under second generation antipsychotics. Obesity (Silver Spring). 2008;16(8):1780-1787.

19. Henderson DC, Cagliero E, Copeland PM, et al. Glucose metabolism in patients with schizophrenia treated with atypical antipsychotic agents: a frequently sampled intravenous glucose tolerance test and minimal model analysis. Arch Gen Psychiatry. 2005;62(1):19-28.

20. Saarni SE, Saarni SI, Fogelholm M, et al. Body composition in psychotic disorders: a general population survey. Psychol Med. 2009;39(5): 801-810.

21. Suvisaari JM, Saarni SI, Perälä J, et al. Metabolic syndrome among persons with schizophrenia and other psychotic disorders in a general population survey. J Clin Psychiatry. 2007;68(7):1045-1055.

22. Roick C, Fritz-Wieacker A, Matschinger H, et al. Health habits of patients with schizophrenia. Soc Psychiatry Psychiatr Epidemiol. 2007; 42(4):268-276.

23. Samele C, Patel M, Boydell J, Leese M, Wessely S, Murray R. Physical illness and lifestyle risk factors in people with their first presentation of psychosis. Soc Psychiatry Psychiatr Epidemiol. 2007;42(2):117-124.

24. McCreadie RG; Scottish Schizophrenia Lifestyle Group. Diet, smoking and cardiovascular risk in people with schizophrenia: descriptive study. Br J Psychiatry. 2003;(183):534-539

25. Amani R. Is dietary pattern of schizophrenia patients different from healthy subjects? BMC Psychiatry. 2007;7:15.

26. Kobayashi S, Honda S, Murakami K, et al. Both comprehensive and brief self-administered diet history questionnaires satisfactorily rank nutrient intakes in Japanese adults. J Epidemiol. 2012;22(2):151-159.
Neuropsychiatric Disease and Treatment

\section{Publish your work in this journal}

Neuropsychiatric Disease and Treatment is an international, peerreviewed journal of clinical therapeutics and pharmacology focusing on concise rapid reporting of clinical or pre-clinical studies on a range of neuropsychiatric and neurological disorders. This journal is indexed on PubMed Central, the 'PsycINFO' database and CAS,

\section{Dovepress}

and is the official journal of The International Neuropsychiatric Association (INA). The manuscript management system is completely online and includes a very quick and fair peer-review system, which is all easy to use. Visit http://www.dovepress.com/testimonials.php to read real quotes from published authors. 